\title{
Dualism of the Heisenberg and Schrödinger Approaches to the Quantum States Entering a One-Dimensional Electron Gas
}

\author{
Stanisław Olszewski \\ Institute of Physical Chemistry, Polish Academy of Sciences Kasprzaka, Warsaw, Poland \\ Email: olsz@ichf.edu.pl
}

How to cite this paper: Olszewski, S. (2020) Dualism of the Heisenberg and Schrödinger Approaches to the Quantum States Entering a One-Dimensional Electron Gas. Journal of Modern Physics, 11, 475-485.

https://doi.org/10.4236/jmp.2020.113030

Received: February 19, 2020

Accepted: March 22, 2020

Published: March 25, 2020

Copyright $\odot 2020$ by author(s) and Scientific Research Publishing Inc. This work is licensed under the Creative Commons Attribution International License (CC BY 4.0).

http://creativecommons.org/licenses/by/4.0/

\begin{abstract}
According to quantum mechanics, the commutation property of the energy Hamiltonian with the momentum operator should give the definite values not only for energy but also for the momentum quantum levels. A difficulty provided by the standing-like boundary conditions of the electron gas is that the Hamiltonian eigenfunctions are different than eigenfunctions of the momentum operator. In results the electron momenta are obtained from the correspondence rule between the classical and quantum mechanics given by Landau and Lifshits. As a consequence the statistics of solutions representing not only the energy values but also the electron momenta should be taken into account. In the Heisenberg picture of quantum mechanics, the momenta are easily obtained because the electron oscillators are there directly considered. In fact, the Hamiltonian entering the Heisenberg method can be defined in two different ways each giving the set of the electron energies known from the Schrödinger's approach.
\end{abstract}

\section{Keywords}

Fundamentals of the Modern Quantum Theory, Heisenberg Picture, Its Momentum Results and the Energy Matrix, Schrödinger Picture and Its Energy Results

\section{Introduction}

Historically we had a competition of the Schrödinger and Heisenberg formalisms in their approach to develop the modern quantum mechanics. Certainly, the Schrödinger method [1] [2] [3] [4] occurred to be more practical in calculating the physical properties of numerous electron systems. But, on the other hand, Heisenberg was the first one who presented foundations of the idea of the 
observables and operators belonging to them [5] [6].

Nevertheless, a practical background of the Heisenberg theory remained rather poor. In fact only the oscillators of different kinds and their properties, especially those concerning the behaviour of the matrix elements, are dominating in the Heisenberg approach.

Our idea is to compare the Heisenberg and Schrödinger methods in examining a very simple system represented by the electrons enclosed in a one-dimensional potential box. To the author's knowledge this comparison seems to have never been done before. A wave-mechanical treatment of such a system performed according to the Schrödinger method occurred to be very simple. On the other hand, the oscillating character and properties of the system remained fully neglected.

An alternative method, suitable for the oscillatory examination, became the Heisenberg theory and an approach to it presented in [7]. The both methods of quantum mechanics could be examined for a non-interacting electron gas case. Physically they indicate a different behaviour of electrons in each kind of the examined theory, as well as in course of their application in statistics.

We note that the Schrödinger's approach to the one-dimensional electron gas can be done in a unique way. On the other hand, the Heisenberg theory could be applied to the same gas in two different ways, each basing on a different Hamiltonian formula used for the same gas object. In consequence the statistics leading to the states occupation by the electrons in the Schrödinger theory should be also slightly changed respectively to the examined Heisenberg's Hamiltonian case.

This is so because of the boundary conditions imposed on electrons entering the Schrödinger model. In effect the electron wave functions represent the standing waves and they are not the eigenfunctions of the momentum operator, contrary to situation due to the Bloch's boundary conditions applied usually to solids. In this second case, however, the requirement of a finite and strictly one-dimensional gas model cannot be satisfied.

A difficulty with the boundary conditions entering the Schrödinger method can be removed when the correspondence rule due to Landau and Lifshits [8] concerning the classical and quantum approaches to the mechanics of electron motion is applied. Because the free-electron Hamiltonian commutes with the operator of the electron momentum, the stationary states of energy should be accompanied by the stationary states of momentum. This is easily obtained on a semi-classical way when the Landau-Lifshits rule is assumed to hold. In effect we should obtain the electron momenta corresponding to the quantum energy levels given by the Schrödinger method, on condition the quantum number $n$ of energy is large. In fact the electron momenta obtained with the aid of the Heisenberg method are found identical with those deduced with the aid of the Schrödinger model also for the low quantum numbers $n$; see Sec. 5. This imposes a question of statistics with which both the energy and momenta of free-electron particles can be satisfactorily considered.

In brief, an alternative method to Schrödinger's-much suitable for the oscillatory examination —is that of Heisenberg; see e.g. [7] [9]. 


\section{Schrödinger's Approach to the Free-Electron Particles}

This approach represents an elementary Schrödinger quantization process and its parameters; see e.g. [10].

Within a one-dimensional potential box of length $L$ we have the free electrons whose energies are defined by the eigenvalues of the Hamiltonian operator:

$$
\hat{H}=-\frac{\hbar^{2}}{2 m_{e}} \frac{\mathrm{d}^{2}}{\mathrm{~d} x^{2}}=\frac{\hat{p}_{x}^{2}}{2 m_{e}} ;
$$

$\hat{p}_{x}$ is the electron momentum operator, $m_{e}$ is the electron mass. In effect we have the eigenequation:

$$
\hat{H} \psi_{n}(x)=E_{n} \psi_{n}(x) .
$$

The wave functions $\psi_{n}(x)$ satisfy the following boundary conditions at the box ends, viz.

$$
\psi_{n}(x=0)=\psi_{n}(x=L)=0 .
$$

Suitable $\psi_{n}(x)$ are easily verified to be

$$
\psi_{n}(x)=\sqrt{\frac{2}{L}} \sin \left(\frac{n \pi}{L} x\right),
$$

the eigenenergies are (see e.g. [10]):

$$
E_{n}=\frac{n^{2} h^{2}}{8 m_{e} L^{2}}
$$

Because of (3) the wave functions (4) are called the standing-like wave functions in the potential box.

\section{Oscillating Character of Electrons Described by $\psi_{n}$ and $E_{n}$}

Free electrons should satisfy the equation

$$
E_{n}=\frac{m_{e}}{2} v_{n}^{2}
$$

where the absolute value of the electron velocity $v_{n}$ is

$$
v_{n}=\left(\frac{2 E_{n}}{m_{e}}\right)^{1 / 2}=\left(\frac{n^{2} h^{2}}{4 m_{e}^{2} L^{2}}\right)^{1 / 2}=\frac{n h}{2 m_{e} L} .
$$

This value of the velocity is expected to be dominating in course of the electron oscillation within the interval

$$
0<x<L
$$

Evidently the electron motion is going from $x=0$ to $x=L$ and vice versa.

The time period $T_{n}$ of the oscillation satisfies the formula

$$
\frac{2 L}{T_{n}}=v_{n},
$$

so 


$$
T_{n}=\frac{2 L}{v_{n}}=2 L \times \frac{2 m_{e} L}{n h}=\frac{4 m_{e} L^{2}}{n h} .
$$

The motion frequency $v$ due to $T_{n}$ is

$$
v_{n}=\frac{1}{T_{n}}=\frac{n h}{4 m_{e} L^{2}} .
$$

In effect the energy provided by $v_{n}$ becomes

$$
E_{n}^{o s c}=h v_{n}=\frac{n h^{2}}{4 m_{e} L^{2}}=\hbar \omega_{n}
$$

so

$$
\omega_{n}=\frac{n h^{2}}{4 m_{e} L^{2}} \frac{1}{\hbar}=\frac{\pi n h}{2 m_{e} L^{2}}
$$

and its reversal is

$$
\frac{1}{\omega_{n}}=\frac{2 m_{e} L^{2}}{\pi n h}
$$

Now our idea is to match results of Sec. 2 with those of Sec. 3.

Before we do such comparison let us note that parameters $T_{\mathrm{n}}$ and $v_{\mathrm{n}}$ satisfy the original Heisenberg relation [5]:

$$
\frac{d}{d n}(n h)=h=\frac{d}{d n} \oint m_{e} \dot{x}^{2} d t
$$

For, from (10) and because of $\dot{x}=v_{n}$ given in (9), we obtain for (14a):

$$
\frac{d}{d n} \oint m_{e} \dot{x}^{2} d t=\frac{d}{d n}\left(m_{e} v_{n}^{2} T_{n}\right)=\frac{d}{d n}\left(m_{e} \frac{4 L^{2}}{T_{n}}\right)=\frac{d}{d n}\left(m_{e} \frac{n h}{m_{e}}\right)=h
$$

which is identical to the result in [8] and (14a).

In the same way we have

$$
\oint p d q=\oint m_{e} \dot{x} d x=m_{e} v_{n} 2 L=m_{e} \frac{(2 L)^{2}}{T_{n}}=m_{e} 4 L^{2} \frac{n h}{4 m_{e} L^{2}}=n h .
$$

If the result in (14c) is considered as the action $J$, it becomes evident that the derivative of energy in (5) done with respect to $J$ provides us with the electron oscillation frequency (11).

\section{Heisenberg Approach Applied to the Electron Oscillators}

In the first step of the Heisenberg approach to the electron gas enclosed in a potential box we consider the Hamiltonian of an oscillator moving in direction of the axis $x$ [7]:

$$
\hat{H}=\frac{\hat{p}_{x}^{2}}{2 m_{e}}+\frac{m_{e} \omega^{2}}{2} x^{2}=m_{e} \omega^{2}\left\|\begin{array}{cccc}
x_{01} x_{10} & 0 & 0 & \cdots \\
0 & x_{01} x_{10}+x_{12} x_{21} & 0 & \cdots \\
0 & 0 & x_{12} x_{21}+x_{23} x_{32} & \cdots \\
\vdots & \vdots & \vdots & \ddots
\end{array}\right\|
$$

where

$$
x_{01} x_{10}=\frac{\hbar}{2 m_{e} \omega},
$$




$$
\begin{aligned}
& x_{12} x_{21}=2 \frac{\hbar}{2 m_{e} \omega}, \\
& x_{23} x_{32}=3 \frac{\hbar}{2 m_{e} \omega}, \\
& x_{34} x_{43}=4 \frac{\hbar}{2 m_{e} \omega},
\end{aligned}
$$

The $x_{m n}$ are the matrix elements of $x$ calculated between the oscillator states $m$ and $n$, the frequencies $\omega$ are those calculated in (13) taken for $n=1$.

When the results in the (16) formulae are substituted to (15) they give the following diagonal elements for the matrix presented in (15):

$$
\begin{gathered}
x_{01} x_{10}=\frac{L^{2}}{2 \pi^{2}}, \\
x_{01} x_{10}+x_{12} x_{21}=(1+2) \frac{L^{2}}{2 \pi^{2}}=\frac{3 L^{2}}{2 \pi^{2}}, \\
x_{12} x_{21}+x_{23} x_{32}=(2+3) \frac{L^{2}}{2 \pi^{2}}=\frac{5 L^{2}}{2 \pi^{2}}, \\
x_{23} x_{32}+x_{34} x_{43}=(3+4) \frac{L^{2}}{2 \pi^{2}}=\frac{7 L^{2}}{2 \pi^{2}},
\end{gathered}
$$

which give the following result for (15):

$$
\hat{H}=m_{e} \omega^{2}\left\|\begin{array}{ccccc}
\frac{L^{2}}{2 \pi^{2}} & 0 & 0 & 0 & \cdots \\
0 & \frac{3 L^{2}}{2 \pi^{2}} & 0 & 0 & \cdots \\
0 & 0 & \frac{5 L^{2}}{2 \pi^{2}} & 0 & \cdots \\
0 & 0 & 0 & \frac{7 L^{2}}{2 \pi^{2}} & \cdots \\
\vdots & \vdots & \vdots & \vdots & \ddots
\end{array}\right\|
$$

Because of $\omega=\omega_{1}$ calculated in (13), the diagonal matrix elements in (18) give the oscillator energies

$$
E_{m}^{o s c}=m_{e} \omega^{2} \frac{L^{2}}{2 \pi^{2}}(2 m+1)=m_{e}\left(\frac{\pi h}{2 m_{e} L^{2}}\right)^{2} \frac{L^{2}}{2 \pi^{2}}(2 m+1)=\frac{h^{2}}{8 m_{e} L^{2}}(2 m+1)
$$

where

$$
m=0,1,2,3, \cdots
$$

Let us note that the oscillator frequency $\omega$ in [7] is considered as a known parameter. In our calculations this frequency is deduced from the electron motion in the potential box; see Sec. 3 .

This feature enables us to present the matrix elements entering the oscillator energy in terms of the matrix elements dependent on the properties characteris 
tic for the motion in the potential box. Typically for the Heisenberg's treatment of an oscillator we choose only a single oscillation frequency $\omega$ for calculating all quantum states. In the Schrödinger picture this frequency is associated with the lowest quantum state $n=1$; see (13).

A passage to the particle energy in the box is very simple. We note that the sums entering the partial traces of the diagonal matrix elements given in (19), viz.

$$
\sum_{m=0}^{m=n-1}(2 m+1)
$$

give respectively

$$
\begin{array}{ll}
n^{2}=1^{2} & \text { for } n=1, \\
n^{2}=1+3=2^{2} & \text { for } n=2, \\
n^{2}=1+3+5=3^{2} & \text { for } n=3, \\
n^{2}=1+3+5+7=4^{2} & \text { for } n=4, \\
n^{2}=1+3+5+7+9=5^{2} & \text { for } n=5,
\end{array}
$$

In effect the (21)-combined with the factor entering the last term in (19) - provide us with the Schrödinger energy results presented in (5).

\section{An alternative Heisenberg Treatment of Free Electrons in the Potential Box}

In this case we apply the Hamiltonian different than in the oscillator case examined in Sec. 4. This kind of treatment takes into account only the kinetic part of the Hamiltonian and neglects the whole of the $x$-dependent (potential) part in the first row of (15):

$$
\hat{H}=\frac{\hat{p}_{x}^{2}}{2 m_{e}} .
$$

Certainly the constant parameters become different than those applied in the Hamiltonian case of Sec. 4.

The first of the diagonal terms of $p_{x}^{2}$ belonging to the energy matrix representing the Hamiltonian in (22) is:

$$
p_{01} p_{10}=x_{01} x_{10} 2 m_{e}^{2} \omega^{2}=\frac{\hbar}{2 m_{e} \omega} 2 m_{e}^{2} \omega^{2}=\frac{\hbar}{2} 2 m_{e} \omega=\frac{h}{2 \pi} m_{e} \frac{\pi h}{2 m_{e} L^{2}}=\frac{h^{2}}{4 L^{2}} .
$$

In fact

$$
p_{01}=m_{e} v_{1}=m_{e} \frac{h}{2 m_{e} L}=\frac{h}{2 L}
$$

if we note that $v_{1}$ entering (24) and

$$
E_{1}=\frac{1}{2 m_{e}} p_{01} p_{10}=\frac{h^{2}}{8 m_{e} L^{2}}
$$

calculated from (23) are equal respectively to $v_{1}$ and $E_{1}$ obtained in the 
Schrödinger theory; see Sec. 3.

The next diagonal element of the matrix $\hat{p}_{x}^{2}$ is

$$
\begin{aligned}
p_{02} p_{20} & =x_{12} x_{21} 2 m_{e}^{2} \omega^{2}=\left(\frac{\hbar}{2 m_{e} \omega}+\frac{2 \hbar}{2 m_{e} \omega}\right) 2 m_{e}^{2} \omega^{2} \\
& =3 \hbar 2 m_{e} \omega=3 \frac{h}{2 \pi} m_{e} \frac{\pi h}{2 m_{e} L^{2}}=\frac{3}{4} \frac{h^{2}}{L^{2}} .
\end{aligned}
$$

When divided by $2 m_{e}$ the expression (26) becomes

$$
\frac{1}{2 m_{e}} p_{02} p_{20}=\frac{3 h^{2}}{8 m_{e} L^{2}} \text {. }
$$

This result added to that obtained in (25) gives

$$
\frac{1}{2 m_{e}} p_{01} p_{10}+\frac{1}{2 m_{e}} p_{02} p_{20}=\frac{4 h^{2}}{8 m_{e} L^{2}}=\frac{2^{2} h^{2}}{8 m_{e} L^{2}}
$$

which is precisely the next Schrödinger value for the free-electron energy, i.e. it is corresponding to the quantum level $n=2$.

If we take the next diagonal term for the matrix of $\hat{p}_{x}^{2}$ which is

$$
\begin{aligned}
p_{03} p_{30} & =\left(\frac{\hbar}{m_{e} \omega}+\frac{3}{2} \frac{\hbar}{m_{e} \omega}\right) 2\left(m_{e} \omega\right)^{2}=\left(1+\frac{3}{2}\right) \hbar 2 m_{e} \omega \\
& =\frac{5 \hbar}{2} 2 m_{e} \omega=5 \frac{h}{2 \pi} m_{e} \frac{\pi h}{2 m_{e} L^{2}}=\frac{5}{4} \frac{h^{2}}{L^{2}},
\end{aligned}
$$

it gives, when multiplied by $\frac{1}{2 m_{e}}$, the result

$$
\frac{1}{2 m_{e}} p_{03} p_{30}=\frac{5}{8} \frac{h^{2}}{m_{e} L^{2}} .
$$

The sum of terms (25), (27) and (30) becomes

$$
\frac{1}{2 m_{e}}\left(p_{01} p_{10}+p_{02} p_{20}+p_{03} p_{30}\right)=\frac{1+3+5}{8 m_{e} L^{2}} h^{2}=\frac{3^{2} h^{2}}{8 m_{e} L^{2}}
$$

which is the Schrödinger energy of a free electron on the level $n=3$.

The procedure can be readily extended to an arbitrary quantum level $n$.

\section{Some Special Statistical Behaviour of the Electron Energy Quanta Present in a One-Dimensional Potential Box}

Till the present point of our considerations we neglected the properties of the electron statistics applied to the electron gas. In fact the problem of the electron spin, and the Pauli exclusion principle connected with it, were not developed enough at the time of an early competition of the Heisenberg and Schrödinger theories. In principle both the boson and fermion statistics seem to be here applicable, first because of the electron oscillation waves considered in the Heisenberg picture, next because of the double spin-dependent occupation of the energy levels connected with the Schrödinger electron gas state. The fermion-like behaviour of electrons seems however to predominate and our task is to make 
only a supplementary insight into the Fermi statistical distribution considered before.

Our point concerns the question whether the highest occupied Fermi level $n_{\max }$ in the one-dimensional gas should be considered as identical with the Fermi energy, or it does represent a distinct energy value. By assuming the second point of view, the Fermi energy-in accordance with former investigations [11] [12] - should be considered as an inflexion point on the Fermi distribution function $F(E)$ plotted as a function of the electron energy $E$.

The function $F(E)$-as it is well known-depends also on the absolute temperature parameter $T$ :

$$
F(E)=\frac{1}{\mathrm{e}^{-E / k T}+1} .
$$

Let us assume $E$ to be an abbreviation of a small value of the difference between the Fermi energy $E_{F}$ and the electron energy on the highest occupied level $n_{\max }$ in the gas which is

$$
E\left(n_{\max }\right)=\frac{h^{2} n_{\max }^{2}}{8 m_{e} L^{2}}
$$

so

$$
E=\Delta E^{(1)}=E_{F}^{(1)}-E^{(1)}\left(n_{\max }\right)>0 .
$$

The superscript (1) indicates that-for simplicity-only the gas having a single electron on each of its energy levels is considered. In principle we assume that $E$ in (34) is a small number.

The first derivative of $F(E)$ in (32) calculated with respect to the energy $E$ gives

$$
\frac{\mathrm{d} F}{\mathrm{~d} E}=\frac{-1}{\left(\mathrm{e}^{-E / k T}+1\right)^{2}}\left(-\frac{1}{k T}\right) \mathrm{e}^{-E / k T}=\frac{\mathrm{e}^{-E / k T}}{\left(e^{-E / k T}+1\right)^{2}} \frac{1}{k T},
$$

whereas the second derivative of $F(E)$ is represented by the derivative of the result obtained in (35):

$$
\begin{aligned}
\frac{\mathrm{d}^{2} F}{\mathrm{~d} E^{2}} & =\frac{1}{k T} \frac{\mathrm{d}}{\mathrm{d} E}\left[\frac{\mathrm{e}^{-E / k T}}{\left(\mathrm{e}^{-E / k T}+1\right)^{2}}\right]=\frac{1}{(k T)^{2}}\left[\frac{2 \mathrm{e}^{-2 E / k T}}{\left(\mathrm{e}^{-E / k T}+1\right)^{3}}-\frac{\mathrm{e}^{-E / k T}}{\left(\mathrm{e}^{-E / k T}+1\right)^{2}}\right] \\
& =\frac{1}{(k T)^{2}} \frac{1}{\left(\mathrm{e}^{-E / k T}+1\right)^{3}}\left[2 \mathrm{e}^{-E / k T}-\mathrm{e}^{-E / k T}\left(\mathrm{e}^{-E / k T}+1\right)\right] \\
& =\frac{1}{(k T)^{2}} \frac{\mathrm{e}^{-E / k T}}{\left(\mathrm{e}^{-E / k T}+1\right)^{3}}\left[2-\mathrm{e}^{-E / k T}-1\right] .
\end{aligned}
$$

The $E$ in the inflexion point should make (36) equal to zero. To attain that it is enough to require the square-bracket to be vanishing

$$
\mathrm{e}^{-E / k T}-1=0
$$

which for small $E / k T$ gives the equation 


$$
1-1+\frac{E}{k T}-\frac{1}{2 !}\left(\frac{E}{k T}\right)^{2} \cong\left(1-\frac{1}{2 !} \frac{E}{k T}\right) \frac{E}{k T}=0 .
$$

The last equation is satisfied when

$$
E=\Delta E^{(1)}=2 ! k T,
$$

so in this case [see (32)]

$$
F\left(E=\Delta E^{(1)}=2 k T\right) \cong \frac{1}{\frac{1}{\mathrm{e}^{2}}+1}
$$

is obtained at the inflexion point.

In the next step we consider a double occupation of the energy levels in the gas by the electrons having an opposite spin. By assuming that

$$
E_{F}^{(2)}=2 E_{F}^{(1)}
$$

and putting for $E$ in (32) the expression

$$
E=\Delta E^{(2)}=2 \Delta E^{(1)},
$$

we obtain the energy $E$ twice as large as $E=\Delta E^{(1)}$.

A substitution of $E=2 \Delta E^{(1)}$ instead of $E=\Delta E^{(1)}$ into Equation (38) gives:

$$
\left(1-\frac{1}{2 !} \frac{2 \Delta E^{(1)}}{k T}\right) \frac{2 \Delta E^{(1)}}{k T}=0
$$

so

$$
\Delta E^{(2)}=2 \Delta E^{(1)}=2 k T .
$$

Therefore the result (44) obtained for a double occupation of the quantum states implies a reduction of $\Delta E^{(1)}$ defined in (39) to a single $k T$.

Both results for $\Delta E^{(1)}$ and $\Delta E^{(2)}$ vanish at $T=0$ giving respectively

$$
E_{F}^{(1)}=E\left(n_{\max }\right)
$$

and

$$
E_{F}^{(2)}=2 E\left(n_{\max }\right)
$$

\section{Possible Duality of Statistics Applied to the Electron Quantum Levels}

A duality of the boson and fermion statistics which can be applied to the electron levels can be detected by examining the energy of the level ensembles obtained in two different ways. For the electron-gas case a better insight seems to be provided by the Schrödinger's method because of its simplicity.

The eigenvalues of the free-electron Hamiltonian considered by Schrödinger (see Sec. 2) are:

$$
E_{n}=\frac{n^{2} h^{2}}{8 m_{e} L^{2}}
$$


where

$$
n=1,2,3, \cdots
$$

giving the results identical to those obtained in (5). These results can be successfully considered with aid of the Fermi-Dirac statistics.

But another approach than that in (46) and (46a) can be obtained for the electrons in a one-dimensional box when instead of the stationary states of the energy Hamiltonian in (1) a spectrum of energies due to the electron oscillations within the potential box is considered.

Because of a free-electron character of the particles we obtain from (11) and (12):

$$
E_{n}^{\mathrm{osc}}=\frac{n h^{2}}{4 m_{e} L^{2}} .
$$

Clasically the electron having the velocity $v_{n}$ undergoes the way $2 L$ along the box in course of the time period $T_{n}$; see (9) and (10).

Evidently the energy in (47) is by a factor of

$$
n / 2
$$

smaller than the energy obtained in (5) and (46). This means that $n / 2$ oscillators are required to provide the energy equal to a single eigenenergy state labeled by a given $n$ indicating a large degeneracy of the oscillator energies necessary for any large $n$.

In effect the energy of a one-dimensional electron gas can be considered also as a superposition of a large number of the boson energy quanta due solely to the electron oscillations.

\section{Summary}

The paper compares two approaches to the energy levels of a free-electron one-dimensional gas done respectively from the point of view of the Heisenberg and Schrödinger quantum theory. This comparison seems to be absent in the literature.

As a starting point we take into account the Schrödinger wave-mechanical calculation which is very simple. In the next step the electrons are considered as oscillators and the Heisenberg matrices are applied. In fact two different kinds of the Heisenberg's Hamiltonian can be examined for free electrons on condition the constant parameters entering the matrices are suitably modified.

A short calculation concerning the position of the Fermi level in the gas as a function of the absolute temperature has been added. It should be noted that the statistics of quantum energy levels presented in both Heisenberg and Schrödinger theories can be different from that valid for the fermions alone.

\section{Conflicts of Interest}

The author declares no conflicts of interest regarding the publication of this paper. 


\section{References}

[1] Schrödinger, E. (1926) Annalen der Physik, 79, 361. https://doi.org/10.1002/andp.19263840404

[2] Schrödinger, E. (1926) Annalen der Physik, 79, 489. https://doi.org/10.1002/andp.19263840602

[3] Schrödinger, E. (1926) Annalen der Physik, 80, 437. https://doi.org/10.1002/andp.19263851302

[4] Schrödinger, E. (1926) Annalen der Physik, 81, 109. https://doi.org/10.1002/andp.19263861802

[5] Heisenberg, W. (1925) Zeitschrift für Physik, 33, 879. https://doi.org/10.1007/BF01328377

[6] Van der Waerden, B.L. (1968) Sources of Quantum Mechanics. Dover Publications, New York. https://doi.org/10.1063/1.3035086

[7] Rubinowicz, W. (1957) Quantum Theory of the Atom. 2nd Edition, Polskie Wydawnictwo Naukowe, Warszawa. (In Polish)

[8] Landau, L.D. and Lifshits, E.M. (1972) Quantum Mechanics. Izdatelstvo Nauka, Moscow. (In Russian)

[9] Schrödinger, E. (1926) Annalen der Physik, 79, 734. https://doi.org/10.1002/andp.19263840804

[10] Eyring, H., Walter, J. and Kimball, G.E. (1957) Quantum Chemistry. 2nd Edition, Wiley, New York.

[11] Slater, J.C. (1967) Quantum Theory of Molecules and Solids. Vol. 3, McGraw-Hill, New York.

[12] Ziman, J.M. (1972) Principles of the Theory of Solids. Cambridge University Press, Cambridge. https://doi.org/10.1017/CBO9781139644075 